\title{
Selected Papers from OECD-NEA PSBT Benchmark
}

\author{
Maria Avramova, ${ }^{1}$ Annalisa Manera, ${ }^{2}$ David Novog, ${ }^{3}$ \\ Diana Cuervo, ${ }^{4}$ and Alessandro Petruzzi ${ }^{5}$ \\ ${ }^{1}$ The Pennsylvania State University, 231 Reber Building, University Park, PA 16802, USA \\ ${ }^{2}$ Department of Engineering \& Radiological Sciences, University of Michigan, 2355 Bonisteel Boulevard, Ann Arbor, \\ MI 48109-2104, USA \\ ${ }^{3}$ McMaster University, 1280 Main Street West, Hamilton, ON, Canada L6K 3N3 \\ ${ }^{4}$ Universidad Politécnica de Madrid (UPM), Avenida Arco de la Victoria 4, 28040 Madrid, Spain \\ ${ }^{5}$ Nuclear Research Group San Piero a Grado, 1291 Via Livornese, San Piero a Grado, 56100 Pisa, Italy
}

Correspondence should be addressed to Annalisa Manera; manera@umich.edu and David Novog; novog@mcmaster.ca

Received 18 April 2013; Accepted 18 April 2013; Published 26 February 2014

Copyright (C) 2014 Maria Avramova et al. This is an open access article distributed under the Creative Commons Attribution License, which permits unrestricted use, distribution, and reproduction in any medium, provided the original work is properly cited.

Historically, the prediction of safety margins has been based on system level thermal-hydraulic calculations employing suitable empirical formulations for assembly specific geometries and fuel-element grid spacers. These works have assessed response, margins, and consequences for the system based on one-dimensional two-fluid or drift-flux type thermalhydraulics formulations with fuel-vendor specific hydraulic losses and heat transfer characteristics for various fuel assemblies, including the so-called hot channel. Analysis of the hot channel gives important information on flow rates, fuel element centerline temperature, fuel sheath temperature, and margin to the departure from nucleate boiling. Given the reliance of the above approaches on empirical formulations obtained from complex and often difficult experiments, there is significant interest in obtaining reliable and accurate results from computation tools which employ more fundamental empirical relationships which can be obtained from subsets of the domain or from other scaled experiments.

The need to refine models for best-estimate calculations, based on good-quality experimental data, has been expressed in many recent meetings in the field of nuclear applications. The needs arising in this respect should not be limited to the currently available macroscopic methods but should be extended to next-generation analysis techniques that focus on more microscopic processes. One of the most valuable databases identified for the thermal-hydraulics modeling was developed by the Nuclear Power Engineering Corporation (NUPEC), Japan, which includes subchannel void fraction and departure from nucleate boiling (DNB) measurements in a representative pressurized water reactor (PWR) fuel assembly. Part of this database is made available for this international benchmark activity entitled as the NUPEC PWR Subchannel and Bundle Tests (PSBT) benchmark. This international project is officially approved by the Japan Ministry of Economy, Trade, and Industry (METI), U.S. Nuclear Regulatory Commission (NRC), and endorsed by the OECD/NEA.

Prediction of local subchannel flows, pressures, temperatures, and other phenomena such as void fraction and critical heat flux $(\mathrm{CHF})$ is becoming increasingly important in the assessment of safety margins for nuclear power plants. In particular the methodologies available for such calculations have seen rapid development over the last ten to fifteen years. These include more mechanistic subchannel analysis codes as well as advanced applications of computational fluid dynamics (CFD). What is interesting is the assessment of the capability of these computational tools to predict the integral effects for experiments performed on whole assemblies under both steady and transient conditions. In this regard, the OECD-NEA has sponsored several international benchmarks pertaining to the thermal-hydraulic phenomena 
in boiling water reactors (published elsewhere) and pressurized water reactors which are documented within this special issue.

Of note also is the relative scarcity in the literature of experimental data covering heat transfer, critical heat flux, and local subchannel void fraction distribution for fullscale assemblies under the same thermalhydraulic conditions as a PWR and utilizing water. For this benchmark Japan Atomic Energy Research Institute (JAERI) has released the experimental data including X-ray computer tomography measurements of the local void fraction for a full-scale PWR assembly at reactor pressures and temperatures. The uniqueness of this benchmark is that it provides insight into prediction capabilities across a wide range of conditions for codes that range from 1-dimensional averaged through to CFD. The fine-mesh high-quality subchannel void fraction and departure from nucleate boiling data encourage advancement in understanding and modeling of complex flow behavior in real bundles. Given the uniqueness and high quality of this database, this benchmark program and its subsequent publications have significantly added to the available scientific literature in this field. The published work in this special issue represents examination using a large number of thermalhydraulic tools ranging from macroscopic system type thermalhydraulic codes down to microscopic examinations using two-phase computational fluid dynamics models.

The papers within the special issue span a wide range of simulation methods. A general review and background of the benchmark activities are provided by M. Avramova et al. Of particular interest here are the comparisons of all the benchmark submissions to one another. In another paper, $M$. Avramova et al. examined the results of the CTF code, a threefluid modification of the COBRA-TF code, and the US NRC code TRACE for the prediction of the PSBT experiments. This paper also includes a discussion of the time offset of $10 \mathrm{~s}$ (developed separately by the CEA in France) which must be applied to the transient portions of the experimental database to account for the heat capacity of the downcomer region in the NUPEC experiment. Further details of the CTF code and the main development issues suggested are included in $\mathrm{M}$. Avramova et al.

The application of the system thermalhydraulic code CATHARE2 to the benchmark is presented by A. Del Nevo et al. Good agreement was found for the 1D results in general; however some overestimation of the central void fraction was observed in the $3 \mathrm{D}$ model results at lower void fractions (below 0.6). M. Valette used the CATHARE3 code which has enhancements on the number of fields treated as well as interfacial area transport capabilities. Simulations were also performed with the $1 \mathrm{D}$ and $3 \mathrm{D}$ capabilities of the code. The $1 \mathrm{D}$ predictions captured the average behavior quite well, and the author notes the limitations in applying the 3D module for subchannel analysis given that it was initially designed to simulate $3 \mathrm{D}$ flows in downcomers and plenums.

K. H. Leung and D. R. Novog used the ASSERT-PV subchannel code to predict the steady-state and transient NUPEC data and also observed overprediction of the subcooled and low quality void fraction and attributed it to the range of pressures being different than the normal application range for ASSERT. Despite these differences, the critical channel power was predicted quite well with the ASSERTPV subchannel code. A further observation of the limitations of the 1995 Groeveneld CHF lookup table at low mass fluxes was also observed. M. Bucci and P. Fillion studied the application of the FLICA-OVAP advanced subchannel code and examined the impact of CHF predictions by using the W3, Shah, and Katto and Ono CHF prediction methods. In these simulations the CHF predictions showed the best agreement with the Shah correlation. The authors also applied a $6 \mathrm{~s}$ delay to temperature transients to account for heat capacity effects. The SUBCHANFLOW, a modernized version of the COBRA subchannel code family, was used by M. Imke and V. H. Sanchez and predicted the subchannel and bundle void fractions very well, with similar small overprediction of void fraction in the low elevation and low void fraction region (similar to many other subchannel and CFD studies in this benchmark-further details on this tendency are provided later). The MATRA subchannel code which uses a mixture formulation was used by D. H. Hwang et al. who determined optimum mixing coefficients for their models. Here the uncertainty in void fraction prediction was also on the same order as the experimental error and the DNB was also most accurately predicted using the 1995 Groeneveld table as opposed to the other mechanistic models they tested. Y. Sung et al. used the VIPRE-W subchannel code, a Westinghouse developed version of the EPRI VIPRE-01 code, and also determined the optimal turbulent mixing coefficient for the NUPEC data. The VIPRE-W predicted void fractions using the modified EPRI mixing models were able to capture the void fraction measurements within the experimental uncertainties.

E. Krepper and R. Rezhek present the results obtained with the CFD code ANSYS CFX-12.1 for the void distribution tests of the benchmark. Relevant aspects of the implemented wall boiling model are reviewed highlighting the uncertainties in several model parameters. It is also shown that the measured cross-sectional averaged values can be reproduced well with a single set of calibrated model parameters for different tests case. The multifield NEPTUNE_CFD code was used to study five selected steady-state subchannel test cases by C. Baudry et al. Simulation results for the void fraction using a standard choice for the physical models and a constant, predetermined bubble diameter are compared to experimental data. Also, calculation results with devoted models for the bubble-size distribution are analyzed showing a visible impact on the subcooled run, giving void fraction closer to experiments than those obtained with a fixed bubble size. I. Kim et al. used the subchannel code FLICA4 and the commercial CFD code STAR-CCM+ in addition to the USNRC system code TRACE to analyze the benchmark experiments. The FLICA results show very good agreement with the experimental results with a small bias which is on the same order as the experimental uncertainty. The CFD results show a slightly larger bias, in particular at low void fractions. Additional details of the CFD calculations using STAR-CCM+ were also provided by S. Lo and J. Osman. 
While the results show very promising prediction capabilities, there remain some key issues for investigation in the future. In particular not all authors reported the details of their prediction methodology and/or if any specific changes were made to the code or input data in order to match the experimental measurements. On one hand, in this regard a blind benchmark may have shed a more realistic view on the capabilities of both codes and analysts. On the other hand, blind benchmarks must undergo rigorous vetting of the specifications such that the participants do not deviate solely based on unplanned distortions in the input scenario. However, throughout the execution phase of this benchmark there were several discussions and clarifications shared amongst the participants that could only have taken place alongside the experimental results. These were mostly discussions centered on the geometry, conditions, and experimental measurements which helped ensure that the analyses were consistent to the greatest extent practicable. These were especially needed given the long period of time since the measurements were conducted and some discrepancies or lack of information in the original NUPEC documents. Hence, while a blind benchmark may have provided additional information, it would not have been practicable in this case.

Several of the computation results for CFD and subchannel analyses within this benchmark also point to overprediction of void fraction at low total void fraction conditions. One explanation is that the X-ray computed tomography measurements included in the benchmark may not efficiently detect very small amounts of nucleate boiling immediately adjacent to the fuel element surface. However, no additional test data is available which can be used to conclusively define the uncertainty in void fraction measurements under these very low void fraction conditions.

The editors would like to thank Dr. Hideaki Utsuno from Japan Nuclear Energy Safety Organization (JNES), Japan; Mr. Alexander Velazquez-Lozada and Dr. Stephen Bajorek from US NRC; and Dr. Enrico Sartori, Dr. Jim Gulliford, and Dr. Akifumi Yamaji from OECD/NEA, whose support, help, contributions, and encouragement in establishing and carrying out this benchmark are invaluable. This benchmark program is the sum of many efforts including the funding agencies and their staff-the Ministry for Economic Trade and Industry (METI), Japan, US NRC, and the Organization of Economic Co-operation and Development (OECD). Special appreciation goes to Professor Kostadin Ivanov from Pennsylvania State University (PSU) for his input into the program. The editors also wish to express their sincere appreciation for the outstanding support offered by the JNES personnel in providing the test data and discussing the test characteristics. Finally, our thanks go to the many authors and researchers who contributed to the technical success of this benchmark.

Maria Avramova

Annalisa Manera David Novog Diana Cuervo

Alessandro Petruzzi 


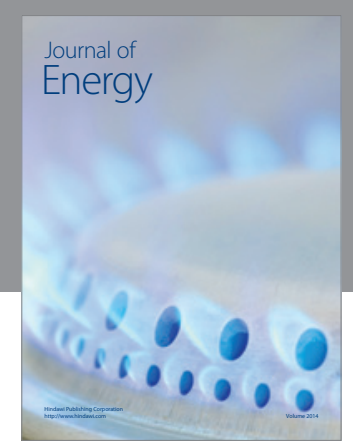

Journal of

Industrial Engineering
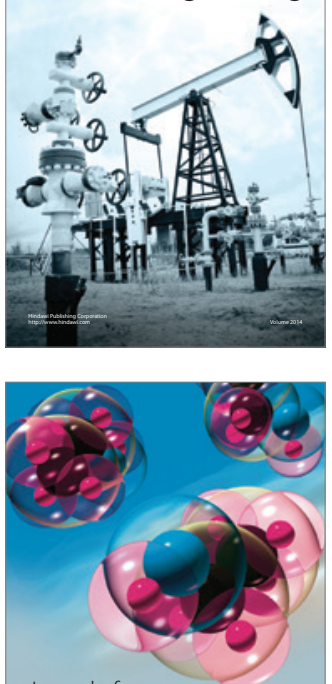

Fuels
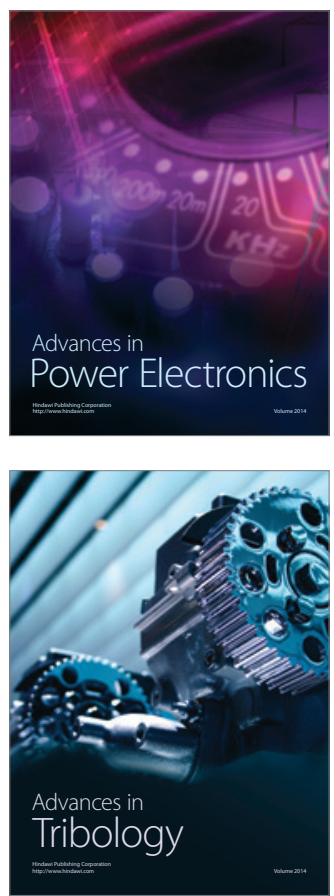

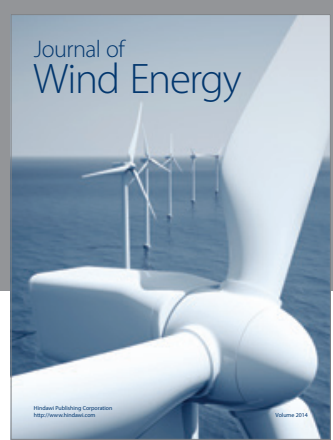

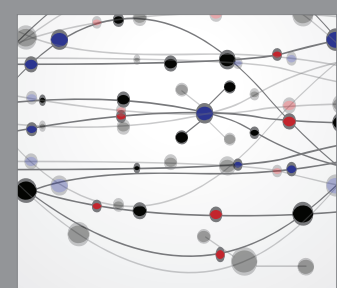

The Scientific World Journal

Submit your manuscripts at http://www.hindawi.com

Journal of

Structures
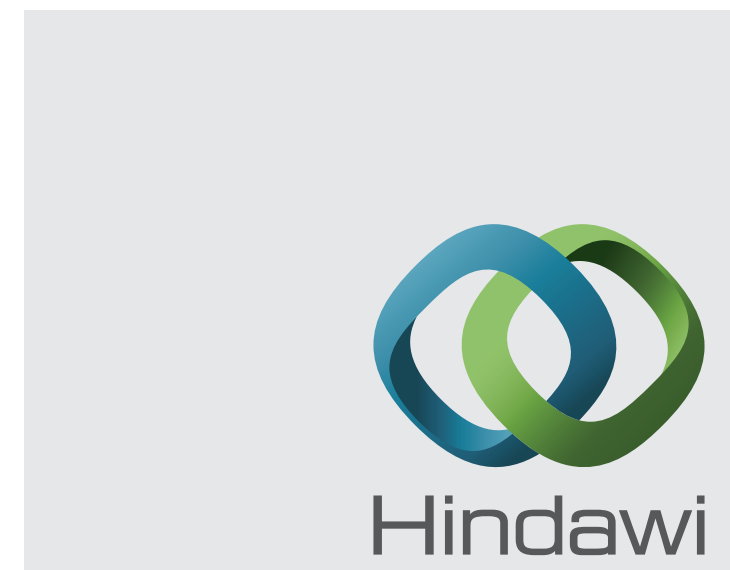

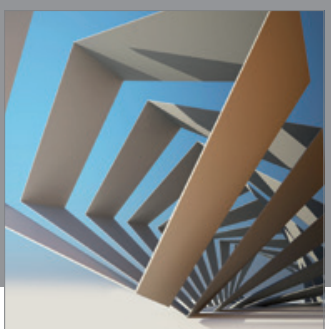

Rotating

Machinery
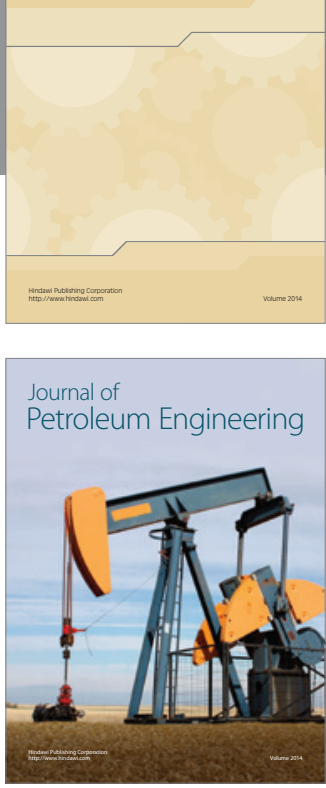

Journal of

Solar Energy
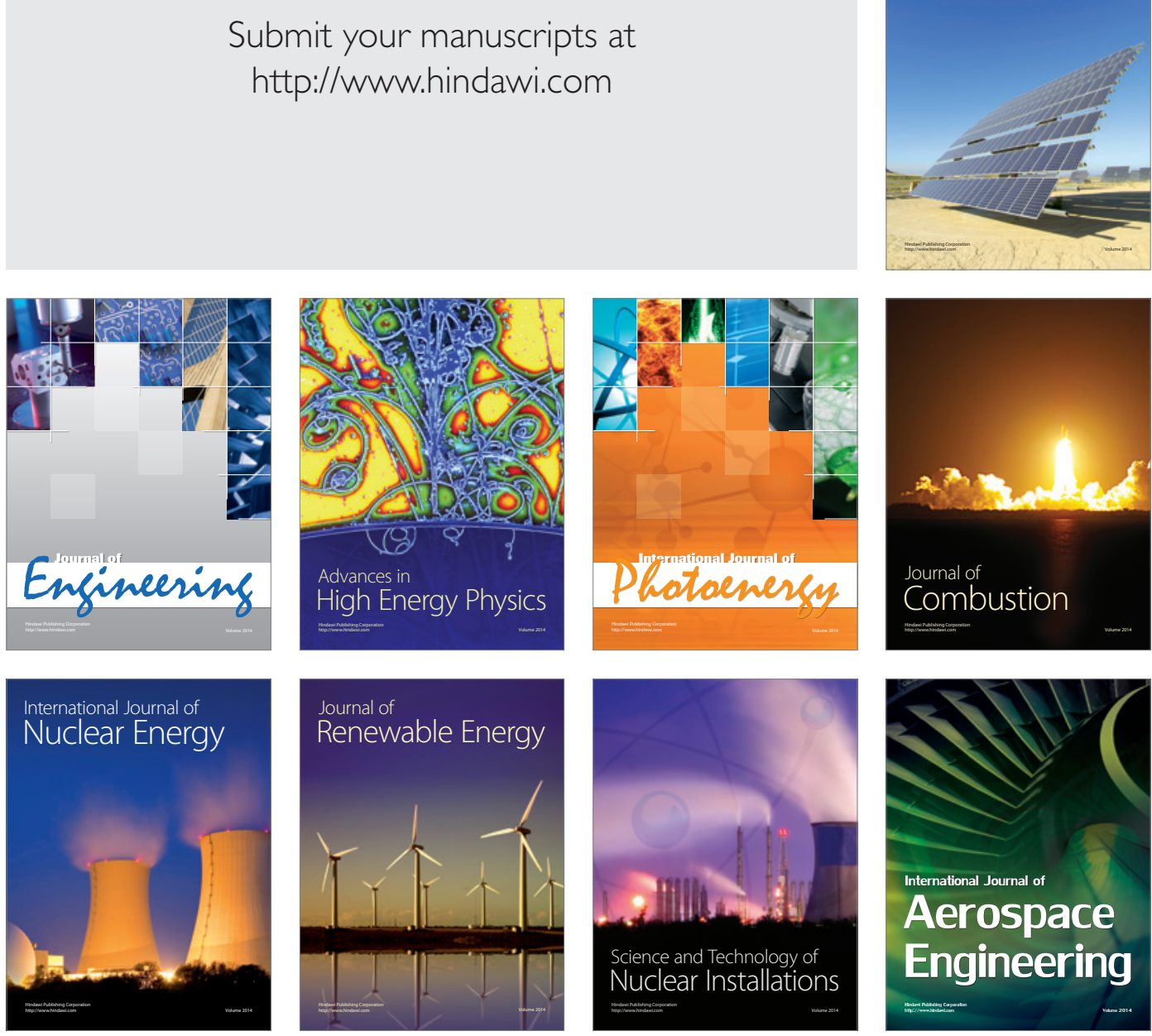